\title{
Local field intensity in aggregates illuminated by diffuse light: $T$ matrix approach
}

\author{
Jean-Claude Auger ${ }^{1}$ and Brian Stout ${ }^{2, *}$ \\ ${ }^{1}$ Center for Laser Diagnostics, Department of Applied Physics, Yale University, New Haven, Connecticut 06520, USA \\ ${ }^{2}$ Institut Fresnel, D.U. Saint Jérôme, Unité Mixte de Recherche 6133, 13397 Marseille Cedex 20, France \\ *Corresponding author: brian.stout@fresnel.fr
}

Received 6 December 2007; revised 24 March 2008; accepted 10 April 2008; posted 16 April 2008 (Doc. ID 90568); published 21 May 2008

\begin{abstract}
We derive an analytic expression for the local field intensity in an aggregate of particles illuminated by diffuse light ( $T$ matrix formalism). To be precise, the diffuse light average is obtained by averaging the electromagnetic response from plane waves over all possible incident field direction polarizations. We applied this new averaging formula to analyze variations in the electromagnetic couplings between two isotropic spheres as a function of their separation distance. The numerical calculations were performed with the recursive centered $T$ matrix algorithm (RCTMA), one of the known analytical solutions of the multiple scattering equation of light. Illustrative calculations clearly demonstrate that diffuse averaging has a large smoothing effect on the strong angular and local variations in the field intensities that are omnipresent in orientation fixed calculations. We believe that this formalism can be a valuable tool in the analysis of electromagnetic couplings in dense random heterogeneous media, in which light propagation is dominated by a scalar diffusion behavior. () 2008 Optical Society of America

OCIS codes: $\quad 260.2110,290.4210,290.5825,290.5850$.
\end{abstract}

\section{Introduction}

During the past decade, the increasing capability to perform accurate and relatively fast numerical evaluations of local fields in structured and disordered heterogeneous systems has played an important role in the advancement of a number of domains of applied physics. Among these, one can site the study of the amplification of the local field intensity in disordered metallic structures where the heterogeneities have sizes much smaller than the wavelength of the incident radiation [1], the analysis of photonic bandgap materials [2], or the effect of the internal field on Raman scattering cross sections [3]. Another interesting domain of application concerns the study of the optical properties of architectural paint films. It is assumed that the analysis of the variation of the local field intensity among the pigments can provide fundamental understanding on the complex electromagnetic couplings that take place in the medium and that are

$0003-6935 / 08 / 162897-09 \$ 15.00 / 0$

(C) 2008 Optical Society of America known to strongly influence the gloss, color, and opacity of the dry films (see, for example, Refs. 四, 5).

Following this line of research, a series of works [6,7] have been carried out on rutile titanium particles, which are used as white pigments in many industries because of their high index of refraction and absence of absorption in the visible range. These simulations [6,7] were performed using the finite difference time domain (FDTD) method [8]. Their results clearly demonstrated strong variations in the near field intensity in function of the direction of propagation and polarization of the incident plane wave as well as the relative positions between the scatterers. However, architectural white paint films, as well as many other materials, are random dense heterogeneous media whose heterogeneities are comparable in size or larger than the wavelengths of the incident radiation. Because light propagation in such media can be relatively well described by the diffusion approximation of the scalar radiative transfer equation (see Ref. [9], for example), such dependencies of the local field intensities on specific polarizations and orientations of the incident radiation are unlikely to 
have a significant effect on overall behavior. Therefore, in order to improve the validity of the fundamental scattering models, one could take into account diffuse illumination.

Because of its wide theoretical range of application, FDTD is certainly one of the most popular techniques currently used to calculate local fields. One of the most cited advantages of this method is to permit the modeling of systems composed of anisotropic materials and to treat arbitrarily shaped particles. Nevertheless, such finite element methods also suffer from a number of nonnegligible disadvantages. First, they necessitate an extensive CPU time, which grows strongly with system size and dimension. Secondly, their numerical implementations are complex, and their development necessitates strong knowledge in computational physics. Finally, to our knowledge, they have been principally adapted to a single coherent incident beam with a fixed direction and orientation with respect to the multiple scattering system. Thus, orientation averaging of both near and far field quantities such as field intensities and cross sections must be evaluated numerically.

An interesting alternative to finite element methods is the multiple scattering centered $T$ matrix formalism introduced by Mackowski [10]. In contrast to FDTD, calculation times do not become longer with increasing distances between the particles, but depend only on the number of scatterers in the system and their relative sizes with respect to the wavelength of the incident field. Furthermore, the local fields can be exactly evaluated at any point in space, whereas in finite element methods, their precision depends on the mesh size and the extrapolation function. An all-important advantage in the present context is that the multiple scattering $T$ matrix formalism can yield analytical expressions for the orientation and polarization averages of both far field optical parameters [11] and the near field averages derived herein.

The aim of this paper is twofold: (a) to propose a theoretical formalism based on the multiple scattering $T$ matrix theory that provides an analytical formulation of the orientation and polarization average of the local electric field intensities and that can be used in structured or randomly configured systems of dielectric or metallic objects and (b) to illustrate possible applications of this formalism through the study of the amplitude of the electromagnetic couplings between two spherical rutile titanium dioxide pigments as a function of their separation distances.

The first part of this work, Section 2 3 , is devoted to theory and is divided into three subsections. In the first two of these subsections, we briefly recall the fundamental expressions related to the multiple scattering $T$ matrix formalism as well as the analytical expressions of the total external field intensity for the case of an incident field with fixed orientation and polarization of the incident field. Building upon our previous work [12], we derive in Section 3 an analytical formulation of the total external electric field intensity under diffuse illumination.
In the second part of this paper, Section 3 , we applied the $T$ matrix formalism to study the variation of the electric field intensity as a function of the distance of separation between two spherical dielectric particles. Two cases are considered: an incident monochromatic plane wave propagating along and perpendicular to the axes connecting the two scatterers, and the same two scatterers under diffuse light illumination. Although a much larger number of particles can readily be treated in this manner, we restricted ourselves to two particles in this work in the interest of simplifying the analysis of the interparticle interactions.

\section{Theory-Multiple Scattering $T$ Matrix and Diffuse Light Formulas}

\section{A. Summary of the Multiple Scattering T-Matrix Method}

We consider a monochromatic plane wave, linearly polarized, incident on an ensemble of $N$ isotropic dielectric objects with complex refractive index $n_{s}^{(j)}$ ( $j$ being the particle label), dispersed in an infinite homogeneous nonabsorbing medium characterized by an index of refraction $n_{m}$. We choose some arbitrary point $O$ as the coordinate system center and label the center of each particle by a position vector $\mathbf{r}_{0}^{(j)}$. For each of the particle centers, we define a local reference frame centered on $\mathbf{r}_{0}^{(j)}$ with axes parallel to the system coordinate system. Thus, any point in space is localized by the position vectors $r$ in the system centered frame or $\mathbf{r}_{j}=\mathbf{r}-\mathbf{r}_{0}^{(j)}$ in a reference frame centered on the $j$ th particle. The incident field has a vacuum wavelength $\lambda$ and wave vector $\mathbf{k}_{i}=k \hat{e}_{k}$, where $k=2 \pi n_{m} / \lambda$, the vector $\mathbf{k}_{i}$ being associated with radial unit vectors $\left(\hat{e}_{k}, \hat{e}_{\theta_{k}}, \hat{e}_{\phi_{k}}\right)$ in a spherical coordinate system. The incident (linear) polarization is specified by $\hat{e}_{i}$ and characterized via a parameter $\gamma \equiv a \cos \left(\hat{e}_{i} \cdot \hat{e}_{\theta_{k}}\right)$. Using appropriately defined vector spherical wave functions (VSWFs) [12], denoted $\Psi$, as basis sets for developing the time harmonic incident $\left(\mathbf{E}_{i}\right)$ and scattered $\left(\mathbf{E}_{s}\right)$ electromagnetic fields, one has

$$
\begin{gathered}
\mathbf{E}_{i}(k \mathbf{r})=E_{0} \operatorname{Rg}\left\{\Psi^{t}(k \mathbf{r})\right\} a_{\gamma}, \\
\mathbf{E}_{s}\left(k \mathbf{r}_{j}\right)=E_{0} \Psi^{t}\left(k \mathbf{r}_{j}\right) f_{N}^{(j)},
\end{gathered}
$$

where $t$ and Rg stand for "transpose of" an "regular part of," whereas $a_{\gamma}$ and $f_{N}^{(j)}$ are column vectors composed of the incident field coefficients and the $j$ th particle scattered field coefficients. The multiple scattering $T$ matrix method takes the form of a linear system of coupled matrix equations [12,[13]:

$$
T_{N}^{(j)}=T_{1}^{(j)}\left[I+\sum_{\substack{l=1 \\ l \neq j}}^{N} H^{(j, l)} T_{N}^{(l)} J^{(l, j)}\right], \quad j=1, \ldots, N
$$

where 
- $I$ is the identity matrix, while $J^{(l, j)}$ and $H^{(j, l)}$ are the regular and irregular translation-addition matrices [14]. They are used to translate field developments on a basis set of spherical vector wave functions around a given origin in terms of spherical vector waves centered on a different origin.

- The symbol $T_{1}^{(j)}$ represents the one-body $T$ matrix of the $j$ th object in the system [15]. When operating on the expansion coefficients of the excitation field impinging upon the particle (denoted $e_{N}^{(j)}$ ), it yields the expansion coefficients $\left(f_{N}^{(j)}\right)$ wave via the relation $f_{N}^{(j)}=T_{1}^{(j)} e_{N}^{(j)}$, with

$$
e_{N}^{(j)}=J^{(j, 0)} a_{\gamma}+\sum_{l=1, l \neq j}^{N} H^{(j, l)} f_{N}^{(l)}
$$

- The $T_{N}^{(j)}$ matrix will be referred to here as the $N$-body $T$ matrix of the $j$ th object in the system. It directly relates the expansion coefficients of the incident field on the entire $N$ particle system to the expansion coefficients, $f_{N}^{(j)}$, of the field scattered by the $j$ th particle via the relation $f_{N}^{(j)}=T_{N}^{(j)} J^{(j, 0)} a_{\gamma}=$ $T_{N}^{(j)} e^{\mathbf{k}_{i} \cdot \mathbf{r}_{0}^{(j)}} a_{\gamma}$ (the last equality being valid when $a_{\gamma}$ are the coefficients of a plane wave with wavenumber $\mathbf{k}_{i}$ ).

Once the $N$-body $T$ matrices are evaluated by solving Eq. (3) [16, 17], analytic expressions can be derived for the total cross sections of the entire system [10]. Although in theory the analytical expressions of the electric fields involve infinite expansions on the VSWFs, in practice it is now well known that an accurate representation of the fields can be achieved when truncating the series to an order $n_{\max }$ that depends on the product of the incident wave number and the size of the particles.

\section{B. Total External Field Expression Under a Fixed Orientation of the Incident Wave}

Applying the superposition principle, the total external electric field, noted $\mathbf{E}_{\text {tot }}$, can be expressed as the sum of the incident wave and the fields scattered by each of the particles. Combining Eqs. (22) and (A1), the latter given in the Appendix, we have

$$
\begin{aligned}
& \mathbf{E}_{\mathrm{tot}}(k \mathbf{r})=E_{0}\left(\hat{e}_{x}, \hat{e}_{y}, \hat{e}_{z}\right)\left[\begin{array}{lll}
\left(E_{i}^{x}\right. & E_{i}^{y} & E_{i}^{z}
\end{array}\right)^{t} \\
& \left.+\sum_{j=1}^{N} C\left(\mathbf{r}_{j}\right) \boldsymbol{\Psi}^{t}\left(k \mathbf{r}_{j}\right) f_{N}^{(j)}\right],
\end{aligned}
$$

where $C\left(\mathbf{r}_{j}\right)$ is the standard dyadic that transforms vectors in the $j$ th particle spherical coordinate basis to the Cartesian coordinate system:

$$
C\left(\mathbf{r}_{j}\right)=\left[\begin{array}{ccc}
\sin \theta_{j} \cos \phi_{j} & \cos \theta_{j} \cos \phi_{j} & -\sin \phi_{j} \\
\sin \theta_{j} \sin \phi_{j} & \cos \theta_{j} \sin \phi_{j} & \cos \phi_{j} \\
\cos \theta_{j} & -\sin \theta_{j} & 0
\end{array}\right] .
$$

$\boldsymbol{\Psi}^{t}\left(k \mathbf{r}_{j}\right)$ are 3 by $2 n_{\max }\left(n_{\max }+2\right)$ matrices that contain the spherical coordinate components of the vector spherical wave functions given in terms of the local spherical coordinate systems. From Eq. (四), the intensity of the total external electric field is simply derived:

$$
\left\|\mathbf{E}_{\mathrm{tot}}(k \mathbf{r})\right\|^{2}=E_{0}^{2}[A+B+C],
$$

with

$$
\begin{gathered}
A=1, B=2 \times \operatorname{Re}\left[\left(E_{i}^{x} E_{i}^{y} E_{i}^{z}\right)^{*} \sum_{j=1}^{N} C\left(\mathbf{r}_{j}\right) \Psi^{t}\left(k \mathbf{r}_{j}\right) f_{N}^{(j)}\right], \\
C=\sum_{j=1}^{N} \sum_{l=1}^{N}\left[f_{N}^{(j)}\right]^{\dagger} \Psi^{*}\left(k \mathbf{r}_{j}\right) C^{t}\left(\mathbf{r}_{j}\right) C\left(\mathbf{r}_{l}\right) \Psi^{t}\left(k \mathbf{r}_{l}\right) f_{N}^{(l)}
\end{gathered}
$$

where $\dagger$ and Re stand for "conjugate transpose of" and "real part of." The formulas in Eq. (66) will prove useful in the derivations that follow, but in practice, it is fastest to calculate the field intensity by first calculating the electric field from Eq. (4) and then simply to take $\mathbf{E}_{\text {tot }}^{*} \cdot \mathbf{E}_{\text {tot }}$. Furthermore, since the $a_{\gamma}$ coefficients are nonnegligible to all multipole orders, it is preferable to use the direct expression for the incident field, Eq. (A1), instead of the expansion on the VSWFs, Eq. (11).

\section{Orientation Average Expression of the Total External Field Intensity}

A considerable advantage of using the $T$ matrix formalism is that the variation in the orientation and polarization of the incident radiation are completely and uniquely contained in the $a_{\gamma}$ expansion coefficients. The averaging process can be broken up into two steps: We first average over all possible directions of the incident field polarization, and second we average over all possible orientations of the wave vector of the incident radiation to simulate diffuse illumination. Taken together, these averaging processes can take the general form

$$
\left\langle a_{\gamma}^{\dagger} M a_{\gamma}\right\rangle_{0}=\frac{\int_{\phi=0}^{2 \pi} \int_{\theta=0}^{\pi} \int_{\gamma=0}^{2 \pi} a_{\gamma}^{\dagger} M a_{\gamma} \mathrm{d} \gamma \sin \theta \mathrm{d} \theta \mathrm{d} \phi \mathrm{d} \gamma}{\int_{\phi=0}^{2 \pi} \int_{\theta=0}^{\pi} \int_{\gamma=0}^{2 \pi} \mathrm{d} \gamma \sin \theta \mathrm{d} \theta \mathrm{d} \phi \mathrm{d} \gamma},
$$

where \langle\rangle$_{0}$ denotes the orientation and polarization averages procedures and $M$ will be a matrix whose coefficients are independent of the integration angles. The average over the polarization angle $\gamma$ corresponds to the average intensity of two perpendicular 
polarizations noted $p$ and $q$ such that

$$
\left\langle a_{\gamma}^{\dagger} M a_{\gamma}\right\rangle_{0}=\frac{1}{2} \frac{1}{4 \pi} \int_{\phi=0}^{2 \pi} \int_{\theta=0}^{\pi}\left[a_{p}^{\dagger} M a_{p}+a_{q}^{\dagger} M a_{q}\right] \sin \theta \mathrm{d} \theta \mathrm{d} \phi .
$$

Then, using the orthonormality of the vector spherical harmonics [18], Eq. (8) leads to

$$
\left\langle a_{\gamma} M a_{\gamma}\right\rangle_{0}=2 \pi \operatorname{Tr}(M),
$$

where $\mathrm{Tr}$ stands for "trace of."

Now, to retrieve a formulation as given in Eq. (77), it is necessary to explicitly introduce the centered $T$ matrices $\tau_{N}^{(j, l)}$, which are related to the $T_{N}^{(j)}$ matrices via the relations

$$
T_{N}^{(j)}=\sum_{l=1}^{N} \tau_{N}^{(j, l)} \boldsymbol{J}^{(l, j)}, \quad j=1, \ldots, N
$$

We have previously established that the calculation of the $\tau_{N}^{(i, j)}$ matrices can be an efficient means of obtaining the $T_{N}^{(i)}$ matrices [11, 12]. Thus, using the representations of the incident and scattered fields' expansions on the VSWFs, the total external field intensity can be expressed as

$$
\begin{aligned}
\left\|\mathbf{E}_{\mathrm{tot}}(k \mathbf{r})\right\|^{2}= & E_{0}^{2} \|\left(\hat{e}_{x}, \hat{e}_{y}, \hat{e}_{z}\right)\left[C(\mathbf{r}) \operatorname{Rg}\left\{\mathbf{\Psi}^{t}(k \mathbf{r})\right\} a_{\gamma}\right. \\
& \left.+\sum_{j=1}^{N} \sum_{l=1}^{N} C\left(\mathbf{r}_{j}\right) \Psi^{t}\left(k \mathbf{r}_{j}\right) \tau_{N}^{(j, l)} \boldsymbol{J}^{(l, 0)} a_{\gamma}\right] \|^{2},
\end{aligned}
$$

which leads to

$$
\begin{aligned}
& \left\|\mathbf{E}_{\text {tot }}(k \mathbf{r})\right\|^{2}=E_{0}^{2}\left[a_{\gamma}^{\dagger} \operatorname{Rg}\left\{\Psi^{*}(k \mathbf{r})\right\} \operatorname{Rg}\left\{\Psi^{t}(k \mathbf{r})\right\} a_{\gamma}\right. \\
& +a_{\gamma}^{\dagger} \operatorname{Rg}\left\{\Psi^{*}(k \mathbf{r})\right\} \sum_{j=1}^{N} \sum_{l=1}^{N} P\left(\hat{\mathbf{r}}, \hat{\mathbf{r}}_{j}\right) \Psi^{t}\left(k \mathbf{r}_{j}\right) \tau_{N}^{(j, l)} \boldsymbol{J}^{(l, 0)} a_{\gamma} \\
& \quad+\sum_{j=1}^{N} \sum_{l=1}^{N} a_{\gamma}^{\dagger} \boldsymbol{J}^{(0, l)}\left[\tau_{N}^{(j, l)}\right]^{\dagger} \Psi^{*}\left(k \mathbf{r}_{j}\right) P\left(\hat{\mathbf{r}}_{j}, \hat{\mathbf{r}}\right) \operatorname{Rg}\left\{\Psi^{t}(k \mathbf{r})\right\} a_{\gamma} \\
& \quad+\sum_{j=1}^{N} \sum_{l=1}^{N} \sum_{i=1}^{N} \sum_{k=1}^{N} a_{\gamma}^{\dagger} \boldsymbol{J}^{(0, k)}\left[\tau_{N}^{(i, k)}\right]^{\dagger} \Psi^{*}\left(k \mathbf{r}_{i}\right) \\
& \left.\quad \times P\left(\hat{\mathbf{r}}_{i}, \hat{\mathbf{r}}_{j}\right) \Psi^{t}\left(k \mathbf{r}_{j}\right) \tau_{N}^{(j, l)} \boldsymbol{J}^{(l, 0)} a_{\gamma}\right]
\end{aligned}
$$

where $P\left(\hat{\mathbf{r}}_{i}, \hat{\mathbf{r}}_{j}\right)=C^{t}\left(\hat{\mathbf{r}}_{i}\right) C\left(\hat{\mathbf{r}}_{j}\right)$. The final stage of the procedure consists of using the translation theorem to express the incident field as a function of the $\boldsymbol{J}^{(l, 0)}$ matrix in the second and third terms of Eq. (12) such that

$$
\mathbf{E}_{i}(k \mathbf{r})=E_{0} C\left(\mathbf{r}_{l}\right) \operatorname{Rg}\left\{\Psi^{t}\left(k \mathbf{r}_{l}\right)\right\} \boldsymbol{J}^{(l, 0)} a_{\gamma} .
$$

Introducing this relation into Eq. (12) leads to

$$
\begin{aligned}
\left\|\mathbf{E}_{\mathrm{tot}}(k \mathbf{r})\right\|^{2}= & E_{0}^{2}\left[\boldsymbol{a}_{\gamma}^{\dagger} \operatorname{Rg}\left\{\boldsymbol{\Psi}^{*}\left(k \mathbf{r}_{l}\right)\right\} \operatorname{Rg}\left\{\boldsymbol{\Psi}^{t}\left(k \mathbf{r}_{j}\right)\right\} a_{\gamma}\right. \\
& +2 \operatorname{Re}\left(\sum_{j=1}^{N} \sum_{l=1}^{N} a_{\gamma}^{\dagger} \boldsymbol{J}^{(0, l)} \operatorname{Rg}\left\{\boldsymbol{\Psi}^{*}\left(k \mathbf{r}_{l}\right)\right\}\right. \\
& \left.\times P\left(\hat{\mathbf{r}}_{l}, \hat{\mathbf{r}}_{j}\right) \boldsymbol{\Psi}^{t}\left(k \mathbf{r}_{j}\right) \tau_{N}^{(j, l)} \boldsymbol{J}^{(l, 0)} a_{\gamma}\right) \\
& +\sum_{j=1}^{N} \sum_{l=1}^{N} \sum_{i=1}^{N} \sum_{k=1}^{N} a_{\gamma}^{\dagger} \boldsymbol{J}^{(0, k)}\left[\tau_{N}^{(i, k)}\right]^{\dagger} \boldsymbol{\Psi}^{*}\left(k \mathbf{r}_{i}\right) \\
& \left.\times P\left(\hat{\mathbf{r}}_{i}, \hat{\mathbf{r}}_{j}\right) \Psi^{t}\left(k \mathbf{r}_{j}\right) \tau_{N}^{(j, l)} \boldsymbol{J}^{(l, 0)} a_{\gamma}\right] .
\end{aligned}
$$

This expression for $\left\|\mathbf{E}_{\text {tot }}\right\|^{2}$ as a later relation has precisely the same form $a_{\gamma}^{\dagger} M a_{\gamma}$ described in Eq. (77). Consequently, applying Eq. (9) to Eq. (14), the orientation average of the total external electric field intensity can be expressed as

$$
\left\langle\left\|\mathbf{E}_{\mathrm{tot}}(k \mathbf{r})\right\|^{2}\right\rangle_{o}=2 \pi E_{0}^{2}\left[A_{o}+B_{o}+C_{o}\right]
$$

with

$$
\begin{aligned}
A_{o}= & 1 / 2 \pi \\
B_{o}= & 2 \operatorname{Re} \operatorname{Tr}\left(\sum_{j=1}^{N} \sum_{l=1}^{N} \operatorname{Rg}\left\{\boldsymbol{\Psi}^{*}\left(k \mathbf{r}_{l}\right)\right\} P\left(\hat{\mathbf{r}}_{l}, \hat{\mathbf{r}}_{j}\right) \Psi^{t}\left(k \mathbf{r}_{j}\right) \tau_{N}^{(j, l)}\right) \\
C_{o}= & \operatorname{Tr}\left(\sum_{j=1}^{N} \sum_{l=1}^{N} \sum_{i=1}^{N} \sum_{k=1}^{N} \boldsymbol{J}^{(l, k)}\left[\tau_{N}^{(i, k)}\right]^{\dagger} \boldsymbol{\Psi}^{*}\left(k \mathbf{r}_{i}\right)\right. \\
& \left.\times P\left(\hat{\mathbf{r}}_{i}, \hat{\mathbf{r}}_{j}\right) \Psi^{t}\left(k \mathbf{r}_{j}\right) \tau_{N}^{(j, l)}\right),
\end{aligned}
$$

where the invariance of the trace on circular permutation and the addition rule of the translation matrices $\boldsymbol{J}^{(l, k)}=\boldsymbol{J}^{(l, 0)} \boldsymbol{J}^{(0, k)}$ and $I=\boldsymbol{J}^{(l, 0)} \boldsymbol{J}^{(0, l)}$ were used. Finally, the exploitation of symmetry relations leads to the final result: 


$$
\begin{aligned}
B_{o} & =2 \times \operatorname{Re} \operatorname{Tr}\left(\sum_{j=1}^{N} \operatorname{Rg}\left\{\Psi^{*}\left(k \mathbf{r}_{j}\right)\right\} \Psi^{t}\left(k \mathbf{r}_{j}\right) \tau_{N}^{(j, j)}\right) \\
& +2 \operatorname{Re} \operatorname{Tr}\left(\sum_{l=1}^{N} \sum_{j>l}^{N} \operatorname{Rg}\left\{\Psi^{*}\left(k \mathbf{r}_{l}\right)\right\} P\left(\hat{\mathbf{r}}_{l}, \hat{\mathbf{r}}_{j}\right) \Psi^{t}\left(k \mathbf{r}_{j}\right) \tau_{N}^{(j, l)}\right)
\end{aligned}
$$

$$
\begin{aligned}
C_{o} & =\operatorname{Tr}\left(\sum_{l=1}^{N} \sum_{i=1}^{N} \sum_{k=1}^{N} \boldsymbol{J}^{(l, k)}\left[\tau_{N}^{(i, k)}\right]^{\dagger} \boldsymbol{\Psi}^{*}\left(k \mathbf{r}_{i}\right) \boldsymbol{\Psi}^{t}\left(k \mathbf{r}_{i}\right) \tau_{N}^{(i, l)}\right) \\
& +\operatorname{Tr}\left(\sum_{i=1}^{N} \sum_{l=1}^{N} \sum_{j>i}^{N} \sum_{k=1}^{N} \boldsymbol{J}^{(l, k)}\right. \\
& \left.\times\left[\tau_{N}^{(i, k)}\right]^{\dagger} \boldsymbol{\Psi}^{*}\left(k \mathbf{r}_{i}\right) P\left(\hat{\mathbf{r}}_{i}, \hat{\mathbf{r}}_{j}\right) \boldsymbol{\Psi}^{t}\left(k \mathbf{r}_{j}\right) \tau_{N}^{(j, l)}\right) .
\end{aligned}
$$

\section{Range of Application}

Although the $T$-matrix formalism is in principle applicable to arbitrarily shaped particles, one needs a reliable method for its calculation. In practice, the Waterman null field approach is most commonly employed, but it usually fails to properly converge for nonaxisymmetric particles. Numerous extensions of the theory have been proposed to overcome this limitation. Some modify the resolution of the surface integrals (see, for example, Ref. [19], or more recently, Ref. [20]), Others use different sets of basis functions [21] and one must convert to the VSWFs before employing Eqs. (15) and (16), and this can apparently can only be done if the smallest circumscribing spheres around each scatterer do not overlap.

Lastly we point out that, contrary to Cruz et al. [22,223, who used the global $T$-matrix approach and consequently were limited to evaluating the local field intensity outside the smallest circumscribing spheres of the entire system, the use of the centered $T$-matrix formalism allows one to readily calculate the field at any point of space outside of the smallest circumscribing sphere of each scatterer [24].

\section{Application}

\section{A. Description of the System Under Study}

The system under study is composed of two isotropic dielectric spheres, noted $S_{1}$ and $S_{2}$, with index of refraction $n_{s}=2.8$, which is known to be a good approximation for anisotropic rutile titanium dioxide crystals. The particles are dispersed in polymer resin $\left(n_{m}=1.5\right)$ and illuminated by a monochromatic plane wave. They have radii $r_{s}=0.12 \times \mu \mathrm{m}$ and are centered at $\left(0,0, z_{1}\right)^{t}$ and $\left(0,0, z_{2}\right)^{t}$ in the principal Cartesian coordinate system. The wavelength of the incident radiation was set to $0.545 \mu \mathrm{m}$, which corresponds to the middle of the visible spectra. Our goal here is to analyze and compare the evolution of the scattering patterns produced by the multiple scattering interactions as the separation distance $\left|z_{2}-z_{1}\right|$ between the objects varies.

To fulfill this objective, the center of $S_{1}$ is fixed at $z_{1}=-0.5 \mu \mathrm{m}$, and we calculate intensity maps of the scattered and total external fields for several different values of $z_{2}$. We focus our attention on the small region of space in the $y \mathrm{Oz}$ plane and that is centered at the origin of the reference frame. The plotting region is a square ranging from -1.0 to $1.0 \times \mu \mathrm{m}$ on the $O y$ and $O z$ axes. Results are displayed in a three dimensional plot with intensity given in the $\mathrm{Oz}$ axis and a contour plot with reversed gray level scale in the upper part of the figure. Calculations were carried out every $5 \times 10^{-3} \times \mu \mathrm{m}$, resulting in a 399 by 399 grid of the observation plane.

\section{B. Fixed Orientations of the Incident Wave Vector Parallel to the $\mathrm{Oz}$ Axis}

In order to compare with the orientation averaged results in Subsection 3.D below, we first applied Eq. (7) to evaluate the total external field intensities resulting from a fixed orientation and two polarizations, transverse electric $(\gamma=\pi / 2)$ and transverse magnetic $(\gamma=0)$ (transverse with respect to the observation plane). Although polarization dependence can be quite strong, for reasons of simplicity we illustrate polarization averaged intensities corresponding to unpolarized incident fields [cf. Eq. (8)]. This first series of calculations were performed while fixing the propagation of the incident plane wave along the positive direction of the $\mathrm{Oz}$ axis. The goal here was to study interparticle coupling effects (i.e., sometimes called dependent scattering), and we found that inclusions of the interference of the incident field with the scattered fields only tended to clutter the analysis. Consequently, we chose to plot the normalized scattered fields intensities $\bar{I}_{s}=I_{s} / I_{0}$. Nevertheless, it seems clear that, were one to study phenomenon such as photonic jets or hot spots, it would be preferable to plot the orientation averaged total field. The intensity map of Fig. 1(a) is that of a sphere $S_{1}$ isolated in the infinite medium, while Figs. [(b) to [1(f) display the intensity patterns of the two particles system for particle separation distances $\left|z_{2}-z_{1}\right|$ equal to $17 r_{s}, 12 r_{s}, 8 r_{s}$, $4 r_{s}$, and $2 r_{s}$, with the incident light directed along the two-particle symmetry axis. Taking the scattering pattern of $S_{1}$ of an isolated sphere as the reference pattern, Figs. [1(b) to [1(f) show a progressive increase in the electromagnetic couplings as the particle separation distance decreases. Such interaction processes can be divided into a succession of qualitatively distinct regions:

- When $\left|z_{2}-z_{1}\right|$ is much larger than the wavelength of the incident field (not shown in the paper), the presence of $S_{2}$ does not significantly affect the scattering pattern near $S_{1}$, leaving unchanged the spatial distribution of the strong intensity in the near field around the particle. Nevertheless, approximately hyperbolic interference patterns appear in the region between the particles. 
- As the separation distance $\left|z_{2}-z_{1}\right|$ decreases to values between ten and several wavelengths of the incident field (Figs. 1(b) 1(d)), the presence of $S_{2}$ has two successive effects on the scattering patterns. In a first step, the curved interference patterns between the particles become more intense and adopt more complex forms. Most notable are the large increases in intensity maxima near the line separating the objects and the strong angular variations of the field intensity around this axis. In a second step, as the particles get considerably closer, the spatial form of the near field intensity is progressively altered, indicating occurrence of strong interparticle couplings.

- When the separation distance $\left|z_{2}-z_{1}\right|$ becomes inferior to a few wavelengths (Fig. $1(\mathrm{e})$ ), the interference pattern in the region between the particles vanishes. The particle $S_{2}$ is now entirely positioned in the near field region of $S_{1}$, resulting in strong nearfield couplings.

- When the two particles are in contact (Fig. [1(f)], the scattering pattern exhibits the characteristics of an isolated nonspherical object in interaction with a monochromatic plane wave.

\section{Fixed Orientations of the Incident Wave Vector Parallel} to the Oy Axis

The particularly strong amplitudes of the interaction between scattered waves illustrated in the previous example is largely due to the specific direction of the incident wave vector that was chosen to be parallel to the axis connecting both scatterers. Thus, to provide an additional source of comparison with diffuse illumination, we mapped the scattered intensity corresponding to an incident wave propagating along the negative $O y$ direction, that is, perpendicular to the axis that binds both scatterers. The interaction process globally shows the same characteristics as described in the preceding subsection. The fact that the scattered intensity of each isolated particle is principally enhanced in the forward direction introduces the following differences with on-axis illumination: (a) the couplings on the $(\mathrm{Oz})$ axes separating the objects are weaker than in the on-axis configuration, and (b) the angular variation around the $O y$ axis, in the half-plane $(z O y)$ and with $y$ negative, are strengthened. Detailed analysis and comparisons between

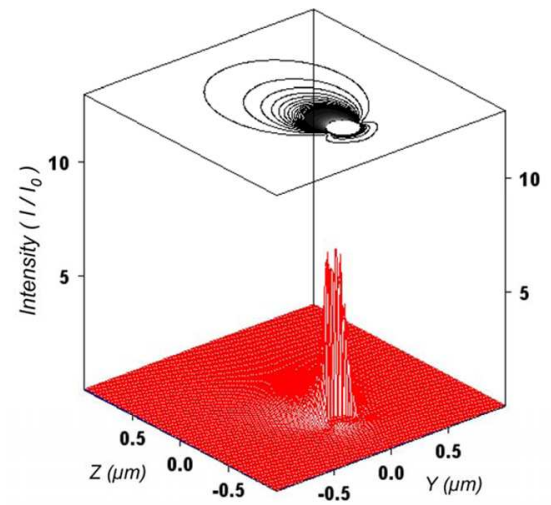

(a)

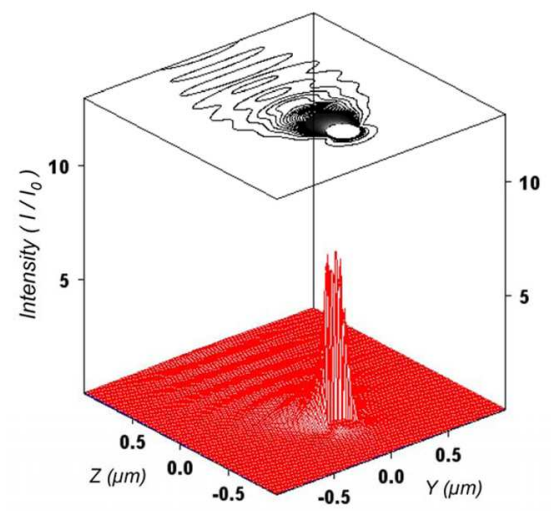

(b)

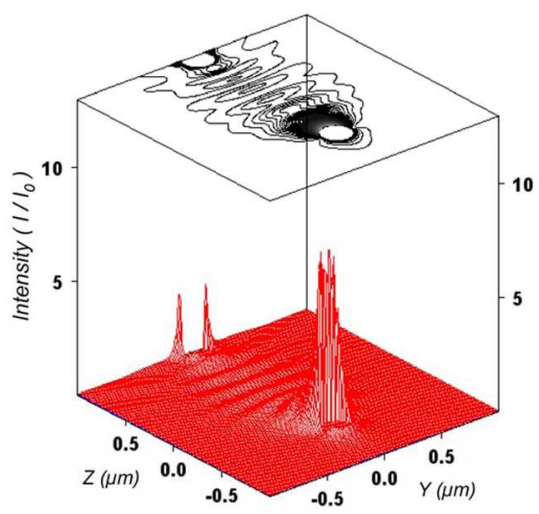

(c)

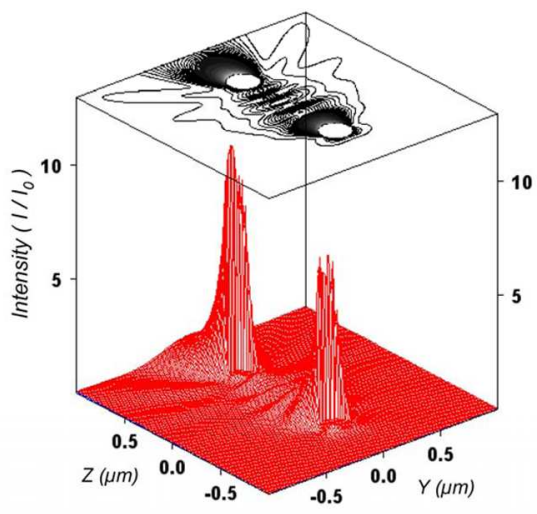

(d)

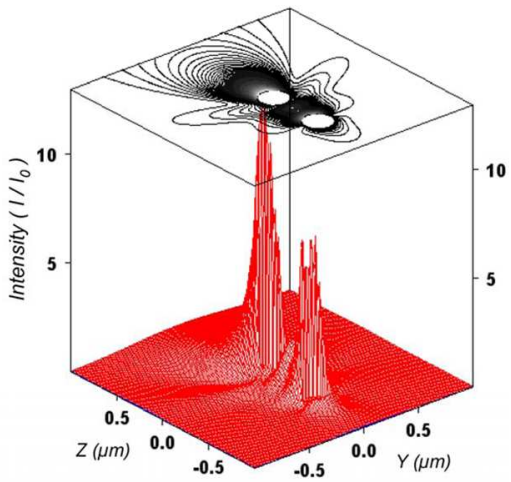

(e)

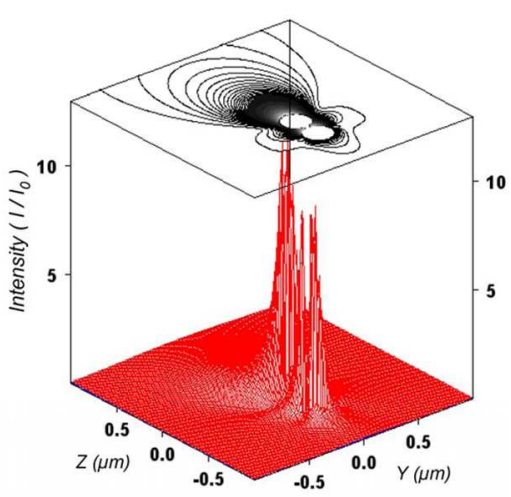

Fig. 1. (Color online) (a) Mapping of the scattered electric field intensity (outside the particles) of a system composed of an isolated dielectric sphere with index of refraction 2.8 and radius $0.12 \mu \mathrm{m}$ embedded into an infinite nonabsorbing medium with refractive index 1.5. The incident field with wavelength of $0.545 \mu \mathrm{m}$ propagates in the positive direction of the $O z$ axis. (b) Same as Fig. $1(\mathrm{a}$ ) but instead the system is composed of two identical dielectric spheres separated by $\left|z_{2}-z_{1}\right|=17 r_{s}$. (c) Same as Fig. 1(b) with separations $\left|z_{2}-z_{1}\right|=12 r_{s}$. (d) Same as Fig. [(b) with separations $\left|z_{2}-z_{1}\right|=8 r_{s}$. (e) Same as Fig. 1(b] with separations $\left|z_{2}-z_{1}\right|=4 r_{s}$. (f) Same as Fig. [1(b) with separations $\left|z_{2}-z_{1}\right|=2 r_{s}$. 


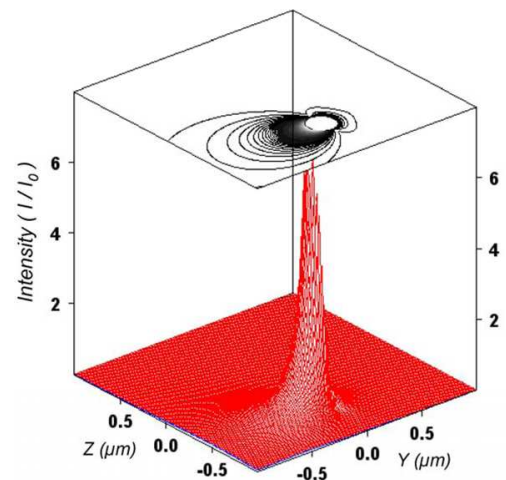

(a)

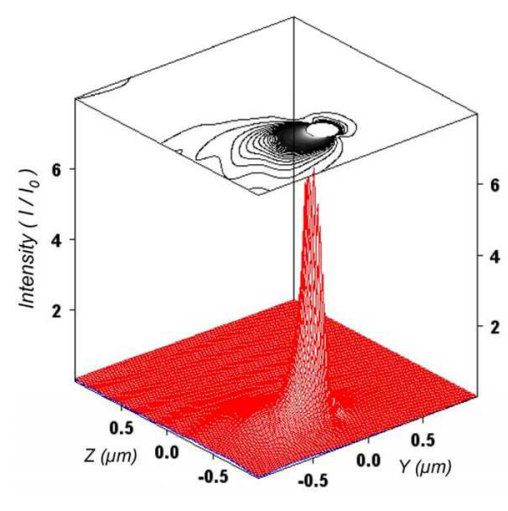

(b)

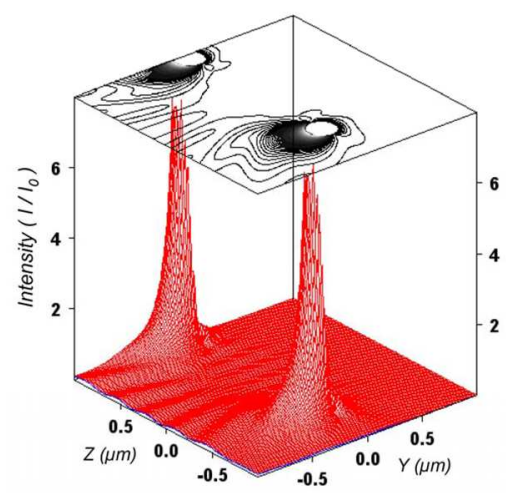

(c)

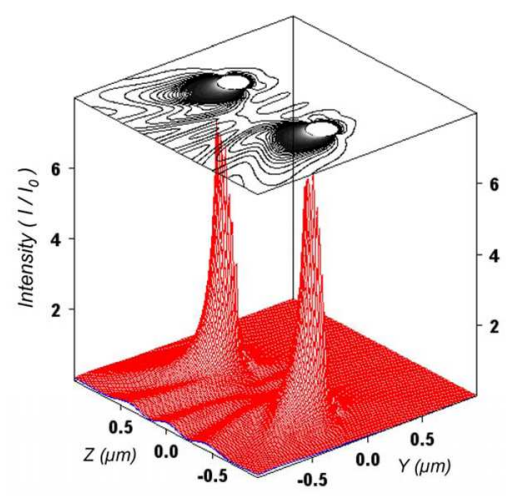

(d)

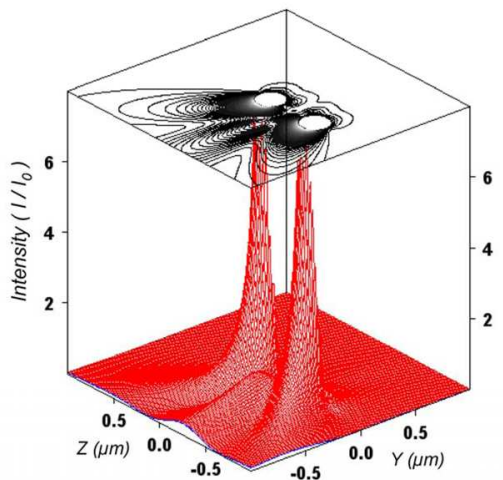

(e)

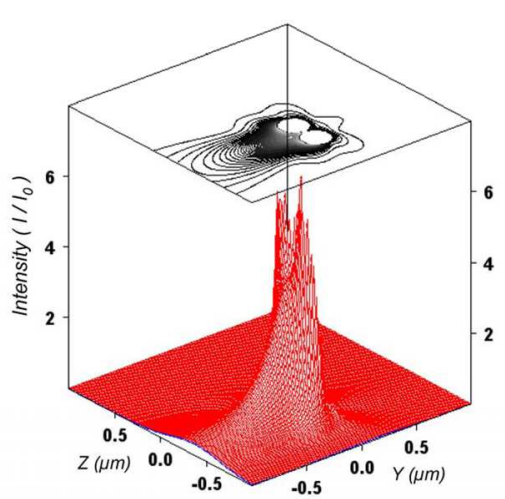

(f)

Fig. 2. (Color online) (a) Mapping of the scattered electric field intensity (outside the particles) of a system composed of an isolated dielectric sphere with index of refraction 2.8 and radius $0.12 \mu \mathrm{m}$ embedded into an infinite nonabsorbing medium with refractive index 1.5. The incident field with wavelength of $0.545 \mu \mathrm{m}$ propagates in the negative direction of the $O y$ axis. (b) Same as Fig. 2(a) but instead the system is composed of two identical dielectric spheres separated by $\left|z_{2}-z_{1}\right|=17 r_{s}$. (c) Same as Fig. 2(a) with separations $\left|z_{2}-z_{1}\right|=12 r_{s}$. (d) Same as Fig. 2(a] with separations $\left|z_{2}-z_{1}\right|=8 r_{s}$. (e) Same as Fig. 2(a) with separations $\left|z_{2}-z_{1}\right|=4 r_{s}$. (f) Same as Fig. 2(a) with separations $\left|z_{2}-z_{1}\right|=2 r_{s}$.

Figs. 1(c) and 2(c), as well as 1(e) and 2(e) clearly show both phenomena.

\section{Diffuse Light Illumination}

To study the effect of diffuse illumination on the electromagnetic couplings, we mapped the normalized orientation average scattered intensity $\left\langle\bar{I}_{s}\right\rangle=\left\langle I_{s}\right\rangle / I_{0}$, in the same system of two dielectric spheres and for the same distances of separation as in Subsections 3.8 and 3.C above. Examination of the resulting cartographies displayed from Fig. 3(a) to Fig. 3(f) shows the following features: (a) a decrease of the couplings in the zone between the particles as compared to the previous systems, (b) the presence of strong field intensity near the particle surfaces, and (c) a drop in the maximum normalized intensity to 2.1 as compared to the fixed orientation enhancements of 12.5 and 10.0 for the on-axis illumination and perpendicular illumination (see Subsections 3.B and 3.C). These observations suggest that the interparticle influence is principally due to couplings of the scattered fields in the zones located near the surface of the scatterers. Indeed, a study of the orientation and averages of the total scattering cross sections only show noticeable differences from the independent scattering re- sults when the surfaces of the particles are separated by less than approximately one diameter.

An important aspect of this study is that the "transitions" between different (independent and dependent) scattering "regimes" are continuous, and one cannot define a precise distance between them. The diffuse scattering results lead us to question the notions of scattering volume around each scatterer as introduced by Fitzwater et al. [25] to describe dependent scattering in white paint films. They argued that at a given concentration, the overlapping of the scattering volumes was the cause of the decrease in the scattering efficiency. Our calculations indicate rather that the dimensions of the scattering volume are not absolute and thereby cannot be readily associated to a threshold in concentration.

\section{Conclusion and Future Work}

Using the recursive centered $T$ matrix formalism, we have derived analytical expressions of the local field intensity when averaging with respect to all directions of the incident monochromatic plane waves and all angles of linear polarization. We applied this formalism to analyze the variations of the local scattered field intensities of a simple system composed of 


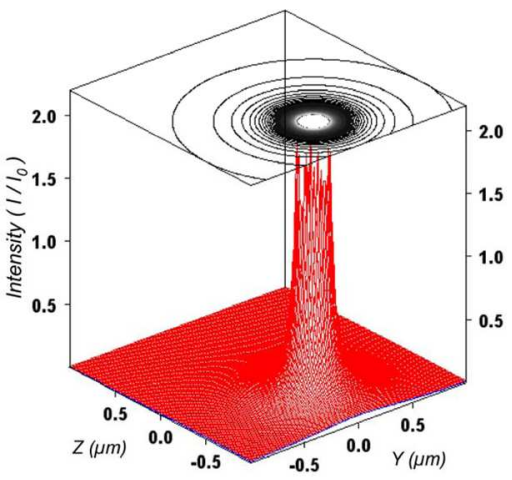

(a)

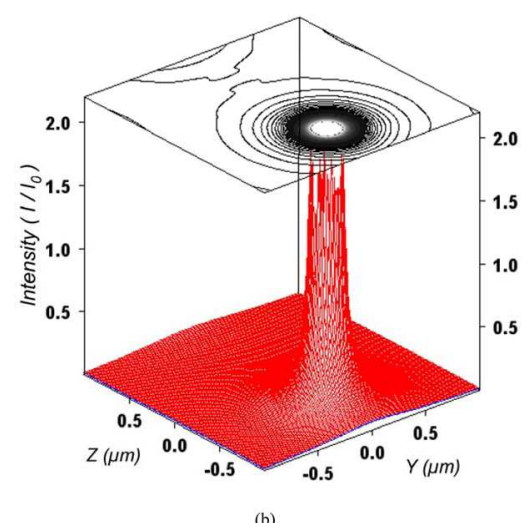

(b)

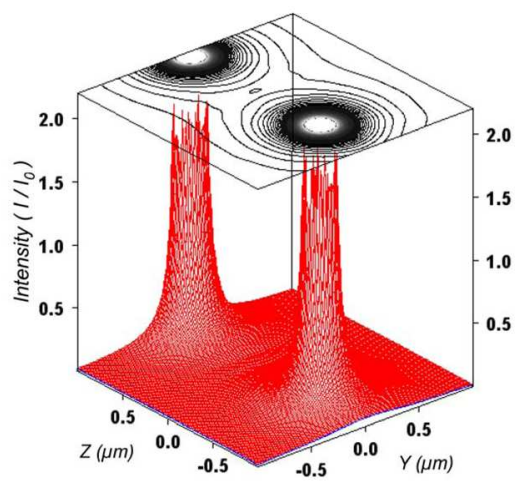

(c)

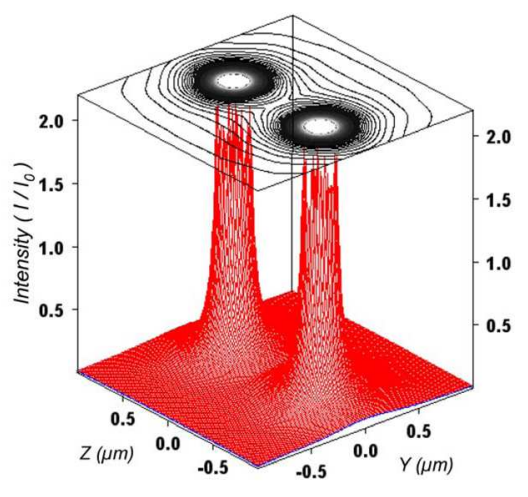

(d)

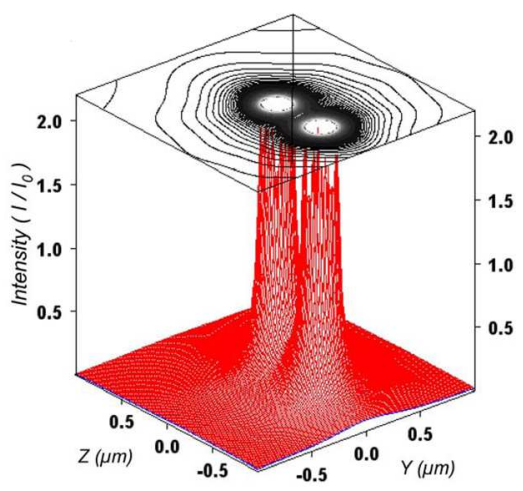

(e)

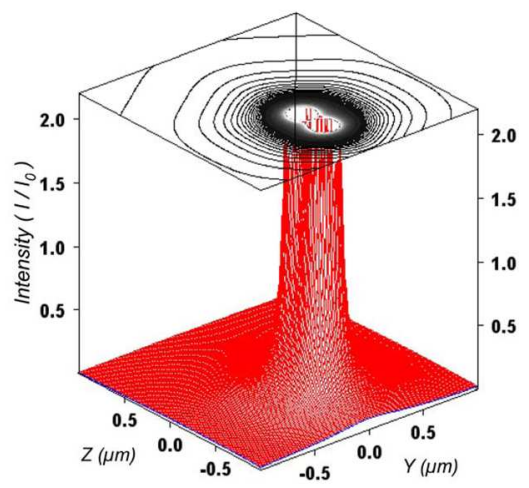

(f)

Fig. 3. (Color online) (a) Mapping of the orientation average scattered electric field intensity (outside the particles) of a system composed of an isolated dielectric sphere with index of refraction 2.8 and radius $0.12 \mu \mathrm{m}$ embedded into an infinite nonabsorbing medium with refractive index 1.5. The wavelength of the incident field is $0.545 \mu \mathrm{m}$. (b) Same as Fig. 3(a) but instead the system is composed of two identical dielectric spheres separated by $\left|z_{2}-z_{1}\right|=17 r_{s}$. (c) Same as Fig. B(a) with separations $\left|z_{2}-z_{1}\right|=12 r_{s}$. (d) Same as Fig. B(a) with separations $\left|z_{2}-z_{1}\right|=8 r_{s}$. (e) Same as Fig. B(a) with separations $\left|z_{2}-z_{1}\right|=4 r_{s}$. (f) Same as Fig. B(a) with separations $\left|z_{2}-z_{1}\right|=2 r_{s}$.

two identical spherical rutile titanium pigments as function of their separation distance.

One direct application would be to compare the angular variation of the local scattered or exciting field intensity around a given scatterer with the far field differential scattered intensity of the whole system after configuration average. Such an analysis would clarify the validity of using far field optical parameters to characterize the volume element of dense heterogeneous media in radiative transfer calculations as is frequently done [26]. Another application could be found in the study of the average local field intensities in clusters of aggregated spheres. Indeed, it has been shown [27] that, for a given wavelength and relative index of refraction, there is a specific size of primary particles at which the orientation and polarization average scattering cross section of the whole cluster is equal to the sum of the individual scattering cross sections in the independent assumption. Studies of the local electric field intensity as a function of the size of the primary particles could provide useful information on the origin of this particular phenomenon.

\section{Appendix A}

The incident plane wave is generally expressed as

$$
\mathbf{E}_{i}(\mathbf{r})=E_{0} \exp \left(i \mathbf{k}_{i} \cdot \mathbf{r}\right) \hat{\mathbf{e}}_{i, \gamma}
$$

In spherical coordinates this reads

$$
\mathbf{E}_{i}(\mathbf{r})=\left[\begin{array}{lll}
\hat{\mathbf{e}}_{k} & \hat{\mathbf{e}}_{\theta_{k}} & \hat{\mathbf{e}}_{\phi_{k}}
\end{array}\right]\left[\begin{array}{c}
0 \\
\cos \gamma \\
\sin \gamma
\end{array}\right] E_{0} \exp \left(i \mathbf{k}_{i} \cdot \mathbf{r}\right) .
$$

In Cartesian coordinates it reads

$$
\begin{aligned}
& \mathbf{E}_{i}(k \mathbf{r})=\left[\begin{array}{lll}
\hat{\mathbf{e}}_{x} & \hat{\mathbf{e}}_{y} & \hat{\mathbf{e}}_{z}
\end{array}\right] \\
& \quad \times\left[\begin{array}{c}
\cos \theta_{k} \cos \phi_{k} \cos \gamma-\sin \phi_{k} \sin \gamma \\
\cos \theta_{k} \sin \phi_{i} \cos \gamma+\cos \phi_{k} \sin \gamma \\
-\sin \theta_{k} \sin \gamma
\end{array}\right] E_{0} \exp \left(i \mathbf{k}_{i} \cdot \mathbf{r}\right)
\end{aligned}
$$


with

$$
\mathbf{k}_{i} \cdot \mathbf{r}=k r\left(\sin \theta_{r} \sin \theta_{k} \cos \left(\phi_{r}-\phi_{k}\right)+\cos \theta_{r} \cos \theta_{k}\right) .
$$

The authors would like to thank R. K. Chang and Eduardo Nahmad for their support given to this work.

\section{References}

1. P. G. Etchegoin, R. C. Maher, and L. F. Cohen, "Amplification of locals in disordered metallic structures," New J. Phys. 6, 142 (2004).

2. A. J. Ward and J. B. Pendry, "Calculating photonic Green's function using a non-orthogonal finite-difference time-domain method," Phys. Rev. B 58, 7252 (1998).

3. J. R. Nestor and E. R. Lippincott, "The effect of internal field on Raman scattering cross sections," J. Raman Spectrosc. 1, 305-318 (2005).

4. R. H. Harding, B. Golding, and R. A. Morgen, "Optics of lightscattering films. Study of effects of pigment size and concentration," J. Opt. Soc. Am. 50, 446-455 (1960).

5. D. F. Tunstall and M. J. Hird, "Effect of particle crowding on scattering power of $\mathrm{TiO}_{2}$ pigments," J. Paint Technol. 46, 3340 (1974).

6. E. S. Thiele and R. H. French, "Light-scattering properties of representative, morphological rutile titanium particles using a finite-element method," J. Am. Ceram. Soc. 81, 469479 (1998).

7. L. E. McNeil, A. R. Hanuska, and R. H. French, "Orientation dependence in near-field scattering from $\mathrm{TiO}_{2}$ particles," Appl. Opt. 40, 3726-3736 (2001).

8. K. S. Kunz and R. J. Luebbers, Finite Difference Time Domain Method for Electromagnetics (CRC Press, 1993).

9. R. Garg, R. K. Prud'homme, and I. A. Aksay, "Optical transmission in highly concentrated dispersion,” J. Opt. Soc. Am. A 15, 932-935 (1998).

10. D. W. Mackowski, "Calculation of total cross sections of multiple-sphere clusters," J. Opt. Soc. Am. A 11, 2851-2861 (1994).

11. B. Stout, J. C. Auger, and J. Lafait, "Individual and aggregate scattering matrices and cross sections: conservation laws and reciprocity," J. Mod. Opt. 48, 2105-2128 (2001).

12. B. Stout, J. C. Auger, and J. Lafait, "A transfer matrix approach to local field calculations in multiple scattering problems," J. Mod. Opt. 49, 2129-2152 (2002).
13. D. W. Mackowski and M. I. Mishchenko, "Calculation of the $T$ matrix and the scattering matrix for ensembles of spheres," J. Opt. Soc. Am. A 13, 2266-2278 (1996).

14. S. Stein, "Addition theorems for spherical wave functions," Q. Appl. Math. 19(1), 15-24 (1961).

15. P. C. Waterman, "Symmetry, unitary and geometry in electromagnetic scattering," Phys. Rev. D 3, 825-839 (1971).

16. A.-K. Hamid, "Electromagnetic scattering by an arbitrary configuration of dielectric spheres," Can. J. Phys. 68, 14191428 (1990).

17. K. A. Fuller and G. W. Kattawar, "Consummate solution to the problem of classical electromagnetic scattering by an ensemble of spheres. I: Linear chains," Opt. Lett. 13, 90-92 (1988).

18. M. I. Mishchenko, L. D. Travis, and A. A. Lacis, Scattering, Absorption and Emission of Light by Small Particles (Cambridge U. Press, 2002).

19. T. Wriedt, "Using the $T$-matrix method for light scattering computations by nonaxisymmetric particles: superellipsoids and realistically shaped particles," Part. Part. Syst. Charact. 19, 256-268 (2002).

20. D. Petrov, Y. Shkuratov, and G. Videen, "Analytical light-scattering solution for Chebyshev particles," J. Opt. Soc. Am. A 24, 1103-1119 (2007).

21. F. M. Schultz, K. Stamnes, and J. J. Stamnes, "Scattering of electromagnetic waves by spheroid particles: a novel approach exploiting the $T$-matrix computed in spheroidal coordinates," Appl. Opt. 37, 7875-7896 (1998).

22. L. Cruz, L. F. Fonseca, and M. Gómez, " $T$-matrix approach for the calculation of local fields in the neighborhood of small clusters in the electrodynamic regime," Phys. Rev. B 40, 74917500 (1989).

23. W. Vargas, L. Cruz, L. F. Fonseca, and M. Gómez, "T-matrix approach for calculation local fields around clusters of rotated spheroids," Appl. Opt. 32, 2164-2170 (1993).

24. J. C. Auger, V. Martinez, and B. Stout, "Absorption and scattering properties of dense ensemble of nonspherical particles," J. Opt. Soc. Am. A 24, 3508-3516 (2007).

25. S. Fitzwater and J. W. Hook III, "Dependent scattering theory: a new approach to predicting scattering in paints," J. Coat. Technol. 57, 39-47 (1985).

26. W. E. Vargas, "Optical properties of pigmented coating taking into account the particle interactions," J. Quant. Spectrosc. Radiat. Transfer 78, 187-195 (2003).

27. J. C. Auger, V. Martinez, and B. Stout, "Scattering efficiency of aggregated clusters of spheres: dependence on configuration and composition," J. Opt. Soc. Am. A 22, 2700-2708 (2005). 